ARTICLE

\title{
Efficient calculation of carrier scattering rates from first principles
}

\author{
Alex M. Ganose (1D ${ }^{1 凶}$, Junsoo Park ${ }^{1}$, Alireza Faghaninia ${ }^{1}$, Rachel Woods-Robinson ${ }^{1,2}$, Kristin A. Persson ${ }^{3,4} \&$ \\ Anubhav Jain (10) ${ }^{1 \times}$
}

The electronic transport behaviour of materials determines their suitability for technological applications. We develop a computationally efficient method for calculating carrier scattering rates of solid-state semiconductors and insulators from first principles inputs. The present method extends existing polar and non-polar electron-phonon coupling, ionized impurity, and piezoelectric scattering mechanisms formulated for isotropic band structures to support highly anisotropic materials. We test the formalism by calculating the electronic transport properties of 23 semiconductors, including the large 48 atom $\mathrm{CH}_{3} \mathrm{NH}_{3} \mathrm{Pbl}_{3}$ hybrid perovskite, and comparing the results against experimental measurements and more detailed scattering simulations. The Spearman rank coefficient of mobility against experiment $\left(r_{\mathrm{s}}=0.93\right)$ improves significantly on results obtained using a constant relaxation time approximation $\left(r_{\mathrm{s}}=0.52\right)$. We find our approach offers similar accuracy to state-of-the art methods at approximately $1 / 500$ th the computational cost, thus enabling its use in high-throughput computational workflows for the accurate screening of carrier mobilities, lifetimes, and thermoelectric power.

\footnotetext{
${ }^{1}$ Energy Technologies Area, Lawrence Berkeley National Laboratory, Berkeley, CA, USA. ${ }^{2}$ Applied Science and Technology Graduate Group, University of California, Berkeley, CA, USA. ${ }^{3}$ Department of Materials Science and Engineering, University of California, Berkeley, CA, USA. ${ }^{4}$ Molecular Foundry, Energy Sciences Area, Lawrence Berkeley National Laboratory, Berkeley, CA, USA. ${ }^{凶}$ email: aganose@lbl.gov; ajain@lbl.gov
} 
S olid-state materials exhibit a variety of electronic transport behaviors, enabling their deployment in a variety of technological applications, including light-emitting devices, photocatalysts, transparent conductors, solar cells, and thermoelectrics ${ }^{1-6}$. Recent years have seen an explosion of interest into the computational prediction of electronic transport properties, leading to a hierarchy of methods that can be broadly split into three categories. (i) Semi-empirical models for approximating electron lifetimes have been employed since the $1930 \mathrm{~s}^{7-12}$ but have seen a resurgence with the advent of large-scale materials science databases due to their computational efficiency ${ }^{13-15}$. These approaches have recently been extended to permit first-principles inputs ${ }^{16-19}$ but the underlying assumption of single parabolic bands with no anisotropy limits their widespread application ${ }^{20}$. (ii) The second category eschews the calculation of electron lifetimes, instead of employing a constant scattering rate for all electronic states. When combined with Fourier $^{21,22}$ or Wannier ${ }^{23}$ interpolation of ab initio electronic band structures this enables efficient calculation of transport properties in complex systems with multiple non-parabolic bands ${ }^{24-26}$. Recent work has applied this approach to compute the transport behavior of large numbers of materials, including 48,000 semiconductors in the Materials Project database by Ricci et al. ${ }^{27}, 809$ sulfides by Miyata et $\mathrm{al}^{28}$, and 75 potential thermoelectric candidates by Xing et al. ${ }^{29}$; however, the unphysical treatment of electron scattering and the reliance on an empirical tuning parameter often results in significant errors. (iii) Finally, the fully first principles approach to calculating the electron-phonon interaction based on density functional perturbation theory (DFPT) combined with Wannier interpolation can now yield highly accurate electron lifetimes and have demonstrated remarkable agreement to experimental measurements of electron mobility and conductivity ${ }^{30-35}$. The calculation of the scattering matrix elements needed to obtain electron lifetimes is highly computationally demanding, even when approximations are made. With few exceptions ${ }^{36-38}$, such approaches have been applied to highly symmetric systems with limited numbers of atoms. ${ }^{39-44}$. Although the computational cost of mobility calculations can be reduced though energy-averaging of the matrix elements ${ }^{45}$, the initial DFPT calculation needed to obtain the matrix elements typically represents the majority of the computational expense. Despite the range of computational techniques available, no existing method can be applied to compute the transport properties of a broad array of complex materials both accurately and inexpensively. This limitation is a primary obstacle in the application of high-throughput computations to the search for novel functional materials as well as applying this theory to larger and more complex materials.

In the present work, we develop an efficient formalism for calculating anisotropic transport properties of semiconductors that is accurate over a range of materials and amenable to use in high-throughput computational workflows. Our approach relies on inputs that can be obtained from low-cost ab initio methods and that are routinely available in computational materials science databases. Scattering rates are calculated using the momentum relaxation time approximation (MRTA) to the Boltzmann transport equation (BTE). The present method includes fully anisotropic acoustic deformation potential, piezoelectric, ionized impurity, and polar electron-phonon scattering. As an initial test of the approach, we calculate the temperaturedependent electron mobility and Seebeck coefficient of 23 semiconductors including the large 48 -atom $\mathrm{CH}_{3} \mathrm{NH}_{3} \mathrm{PbI}_{3}$ hybrid perovskite. The Spearman rank coefficient of mobility against experiment $\left(r_{\mathrm{s}}=0.93\right)$ improves significantly on results obtained using a constant relaxation time approximation $\left(r_{\mathrm{s}}=0.52\right)$. Furthermore, we find our approach offers similar accuracy to stateof-the art methods at 1/500th the computational cost. An open source software implementation of the method is made freely available.

\section{Results}

Computationally efficient matrix elements. The scattering rate of an electron from an initial state $n \mathbf{k}$, where $n$ is a band index and $\mathbf{k}$ is a wave vector, to final state $m \mathbf{k}+\mathbf{q}$ is described by Fermi's golden rule as

$$
\tau_{n \mathbf{k} \rightarrow m \mathbf{k}+\mathbf{q}}^{-1}=\frac{2 \pi}{\hbar}\left|g_{n m}(\mathbf{k}, \mathbf{q})\right|^{2} \delta\left(\varepsilon_{n \mathbf{k}}-\varepsilon_{m \mathbf{k}+\mathbf{q}}\right)
$$

where $\hbar$ is the reduced Planck's constant, $\varepsilon$ is the electron energy, $\delta$ is the Dirac delta function and $g$ is the coupling matrix element. The above equation is given for the case of perfectly elastic scattering ${ }^{46}$, in which electrons do not gain or lose energy during the scattering process. A similar equation can be defined for inelastic processes (Supplementary Eq. 7), for instance to describe scattering that occurs via emission or absorption of a phonon. In general, however, the impact of different scattering mechanisms is expressed via the coupling matrix element $g_{n m}(\mathbf{k}, \mathbf{q})=\langle m \mathbf{k}+$ $\left.\mathbf{q}\left|\Delta_{\mathbf{q}} V\right| n \mathbf{k}\right\rangle$ where $\Delta_{\mathbf{q}} V$ is an electronic perturbation of some kind. The primary obstacle in obtaining accurate transport properties is evaluating $g_{n m}(\mathbf{k}, \mathbf{q})$ on dense Brillouin zone grids, which has so far proven computationally prohibitive for all but the simplest systems $^{47,48}$.

Historically, this challenge has been avoided. In the constant relaxation time approximation (CRTA), Eq. (1) is simplified to a single constant. An alternative is to employ model matrix elements formulated for isotropic band structures based on intrinsic materials parameters. For example, the treatment of deformation potential scattering due to long-wavelength acoustic phonons proposed by Bardeen and Shockley ${ }^{8}$ depends only on an averaged elastic constant and band edge deformation potential; it ignores perturbations from transverse phonon modes and anisotropy in the deformation response. This simple approach has been employed widely in computations of acoustic phonon scattering but is unreliable and does not generalise to complex systems or metals ${ }^{49-51}$. An alternative approach, developed by Khan and Allen ${ }^{49}$, can reproduce the fully first principles electron-phonon scattering rate if the strain tensor caused by the phonon and an additional velocity term are included. The resulting matrix element is given by

$$
g_{n m}^{\mathrm{KA}}=\left\langle m \mathbf{k}+\mathbf{q}\left|\mathbf{S}_{\mathbf{q}}:\left(\mathbf{D}_{n \mathbf{k}}+\mathbf{v}_{n \mathbf{k}} \otimes \mathbf{v}_{n \mathbf{k}}\right)\right| n \mathbf{k}\right\rangle,
$$

where : denotes the double dot product, $\mathbf{S}_{\mathbf{q}}$ is the strain associated with an acoustic phonon, $\mathbf{D}_{n \mathbf{k}}$ is the second rank deformation potential tensor and $\mathbf{v}_{n \mathbf{k}}$ is the group velocity. The velocity term is essential to correct the deformation potential in metals and at states away from the valence or conduction band edge in semiconductors. In practice, however, this equation is no longer simple to evaluate as it requires knowledge of the atomic displacements (the polarization direction) of the phonon mode in order to obtain the strain tensor.

In the present work, we combine the simplicity of the BardeenShockley approach with the accuracy of the Khan-Allen matrix element by exploiting the acoustoelastic properties of materials. The dispersion relations for acoustic waves are contained in the Christoffel equation ${ }^{52}$

$$
\left[\Gamma_{\hat{\mathbf{q}}}-\rho c^{2} \mathbb{1}\right] \hat{\mathbf{u}}=0,
$$

where 1 is the identity matrix, $\hat{\mathbf{q}}$ and $\hat{\mathbf{u}}$ are unit vectors giving the direction of phonon propagation and polarization, respectively, $\rho$ is the density, $c$ is the wave velocity, and $\Gamma_{\hat{\mathbf{q}}}=\mathbf{C} \hat{\mathbf{q}} \cdot \hat{\mathbf{q}}$ is the Christoffel matrix where $\mathbf{C}$ is the rank 4 elastic constant tensor. 
Solving the Christoffel equation for a phonon wave vector direction $(\hat{\mathbf{q}})$ results in three sets of eigenvalues $\left(\rho c^{2}\right)$ and eigenvectors ( $(\hat{\mathbf{u}})$, that correspond to the (quasi-)longitudinal and (quasi-)transverse normal modes of the material. The unit strain associated with each mode is given by $\hat{\mathbf{S}}=\hat{\mathbf{q}} \otimes \hat{\mathbf{u}}$ and the amplitude of the strain at any temperature $T$ can be obtained from the potential energy of the acoustic phonon as $\sqrt{k_{\mathrm{B}} T / \rho c^{2}}$, where $k_{\mathrm{B}}$ is the Boltzmann constant ${ }^{53}$. From this we arrive at an expression for acoustic deformation potential scattering (ad) that relies only on the deformation potentials and elastic constants and includes scattering from longitudinal and transverse modes in a single matrix element, given in the Born approximation ${ }^{54}$ as

$$
g_{n m}^{\mathrm{ad}}(\mathbf{k}, \mathbf{q})=\sqrt{k_{\mathrm{B}} T} \sum_{\mathbf{G} \neq-\mathbf{q}}\left[\frac{\tilde{\mathbf{D}}_{n \mathbf{k}}: \hat{\mathbf{S}}_{l}}{c_{l} \sqrt{\rho}}+\frac{\tilde{\mathbf{D}}_{n \mathbf{k}}: \hat{\mathbf{S}}_{t_{1}}}{c_{t_{1}} \sqrt{\rho}}+\frac{\tilde{\mathbf{D}}_{n \mathbf{k}}: \hat{\mathbf{S}}_{t_{2}}}{c_{t_{2}} \sqrt{\rho}}\right]\left\langle m \mathbf{k}+\mathbf{q}\left|e^{i(\mathbf{q}+\mathbf{G}) \cdot \mathbf{r}}\right| n \mathbf{k}\right\rangle
$$

where $\tilde{\mathbf{D}}_{n \mathbf{k}}=\mathbf{D}_{n \mathbf{k}}+\mathbf{v}_{n \mathbf{k}} \otimes \mathbf{v}_{n \mathbf{k}}$, and the subscripts $l, t_{1}$, and $t_{2}$ indicate properties belonging to the longitudinal and transverse modes.

Scattering by acoustic phonons through the piezoelectric interaction (pi) occurs in non-centrosymmetric systems and can dominate at low temperatures $(\lesssim 50 \mathrm{~K})$. We have applied a similar treatment to extend the isotropic matrix element of Meijer and Polder $^{11}$, Harrison ${ }^{55}$, and Zook ${ }^{53}$, to include the full piezoelectric stress tensor $\mathbf{h}$ and scattering from all three acoustic modes. The resulting matrix element is given by

$$
g_{n m}^{\mathrm{pi}}(\mathbf{k}, \mathbf{q})=\sqrt{k_{\mathrm{B}} T} \sum_{\mathbf{G} \neq-\mathbf{q}}\left[\frac{\hat{\mathbf{n}} \mathbf{\mathbf { h }}: \hat{\mathbf{S}}_{l}}{c_{l} \sqrt{\rho}}+\frac{\hat{\mathbf{n h}}: \hat{\mathbf{S}}_{t_{1}}}{c_{t_{1}} \sqrt{\rho}}+\frac{\hat{\mathbf{h}} \mathbf{h} \hat{\mathbf{S}}_{t_{2}}}{c_{t_{2}} \sqrt{\rho}}\right] \frac{\left\langle m \mathbf{k}+\mathbf{q}\left|e^{i(\mathbf{q}+\mathbf{G}) \cdot \mathbf{r}}\right| n \mathbf{k}\right\rangle}{|\mathbf{q}+\mathbf{G}|},
$$

where $\hat{\mathbf{n}}=(\mathbf{q}+\mathbf{G}) /|\mathbf{q}+\mathbf{G}|$ is a unit vector in the direction of scattering. Due to the small energies of long-wavelength acoustic phonons, both piezoelectric and acoustic deformation potential scattering describe a purely elastic process.

We treat polar optical phonon scattering (po) by extending the Frölich model ${ }^{12}$ to include quantum mechanical wave function overlaps and anisotropic permittivity. Here, electrons in a dielectric medium are perturbed by a dispersionless longitudinal optical phonon mode with frequency $\omega_{\mathrm{po}}$. Our electron-phonon matrix element takes the form

$$
\begin{aligned}
g_{n m}^{\mathrm{po}}(\mathbf{k}, \mathbf{q})= & {\left[\frac{\hbar \omega_{\mathrm{po}}}{2}\right]^{1 / 2} \sum_{\mathbf{G} \neq-\mathbf{q}}\left(\frac{1}{\hat{\mathbf{n}} \cdot \boldsymbol{\epsilon}_{\infty} \cdot \hat{\mathbf{n}}}-\frac{1}{\hat{\mathbf{n}} \cdot \boldsymbol{\epsilon}_{\mathrm{s}} \cdot \hat{\mathbf{n}}}\right)^{1 / 2} } \\
& \times \frac{\left\langle m \mathbf{k}+\mathbf{q}\left|e^{i(\mathbf{q}+\mathbf{G}) \cdot \mathbf{r}}\right| n \mathbf{k}\right\rangle}{|\mathbf{q}+\mathbf{G}|}
\end{aligned}
$$

where $\boldsymbol{\epsilon}_{\mathrm{s}}$ and $\boldsymbol{\epsilon}_{\infty}$ are the static and high-frequency dielectric tensors. To capture scattering from the full phonon band structure in a single phonon frequency, each phonon mode is weighted by the dipole moment it produces (Supplementary Eq. 29) in line with recent work that has rederived the Frölich model for systems with multiple phonon branches ${ }^{56,57}$. Both our extension of the Frölich model and state-of-the-art first principles approaches produce similar matrix elements in the longwavelength limit that dominates scattering (due to the polar singularity at $\mathbf{q} \rightarrow 0^{56}$ ).

Following the classic treatment of Brooks and Herring ${ }^{9,58}$ we consider the scattering from fully ionized impurities (ii) modeled as screened Coulomb potentials, with the matrix element given by

$$
g_{n m}^{\mathrm{ii}}(\mathbf{k}, \mathbf{q})=\sum_{\mathbf{G} \neq-\mathbf{q}} \frac{n_{\mathrm{ii}}^{1 / 2} Z e}{\hat{\mathbf{n}} \cdot \boldsymbol{\epsilon}_{\mathrm{s}} \cdot \hat{\mathbf{n}}} \frac{\left\langle m \mathbf{k}+\mathbf{q}\left|e^{i(\mathbf{q}+\mathbf{G}) \cdot \mathbf{r}}\right| n \mathbf{k}\right\rangle}{|\mathbf{q}+\mathbf{G}|^{2}+\beta^{2}},
$$

where $Z$ is the charge state of the impurity center, $e$ is the electron charge, $n_{\mathrm{ii}}=\left(n_{\mathrm{h}}-n_{\mathrm{e}}\right) / Z$ is the concentration of ionized impurities, and $\beta$ is the inverse screening length (Supplementary Eq. 10). Unlike previous formulations, our matrix element accounts for anisotropy in the charge screening through use of the full dielectric tensor. Taken together, Eqs. (1) and (7) reveal that, assuming weak screening, the scattering almost diverges at long wavelengths $(\mathbf{q} \rightarrow 0)$ due to a $1 /|\mathbf{q}|^{4}$ dependence, and therefore requires very fine sampling to describe correctly. For this reason, almost all attempts at calculating electron scattering by ionized impurities have employed the Brooks-Herring formula, in which Eq. (7) is analytically integrated over the phase space for an isotropic parabolic band ${ }^{32,59}$. To overcome this limitation, we employ a modified linear-tetrahedron approach to integration, in which tetrahedron cross sections are numerically resampled with hundreds of extra points that exactly satisfy the delta term in Eq. (1). This allows for effective k-point mesh densities that would be almost impossible to achieve with uniform k-point sampling (the full methodology is provided in the Supplementary Methods). While other works have used similar matrix elements ${ }^{60}$, we extend these approaches by considering interband scattering and first-principles wave function overlaps in the evaluation of Coulomb-based impurity scattering. In Supplementary Fig. 5, we demonstrate that our methodology reproduces the exact Brooks-Herring mobility for parabolic band structures and reveal the failure of the Brooks-Herring approach in the case of systems containing multiple anisotropic valleys.

The final k-dependent scattering rates are obtained by integrating Eq. (1) over all phonon wave vectors (q) in the first Brillouin zone. Elastic scattering processes are well described by the MRTA to the BTE due to the requirement that $\tau_{n \mathbf{k} \rightarrow m \mathbf{k}+\mathbf{q}}=\tau_{m \mathbf{k}+\mathbf{q} \rightarrow n \mathbf{k}}{ }^{40}$. As this condition does not hold for inelastic processes, we adopt the selfenergy relaxation time approximation (SERTA) to obtain the final polar phonon coupling rates ${ }^{32}$. Further justification for this approach is detailed in the Supplementary Methods. Electronic eigenvalues and group velocities needed to calculate scattering and transport properties are Fourier interpolated onto dense Brillouin zone grids using the BoltzTraP2 software ${ }^{22}$ (as detailed in the Supplementary Methods). Electron mobility and Seebeck coefficient are calculated using the linearized BTE via the Onsager transport coefficients $^{22,61}$ (Supplementary Eqs. 13 to 15). We also employ a custom procedure for selecting the most important $\mathbf{k}$-points at which to calculate scattering to further reduce the computational expense (detailed in the Supplementary Methods).

Unlike other state-of-the-art approaches in which a computationally expensive DFPT calculation is required to obtain $g(\mathbf{k}, \mathbf{q})$, in our method all matrix elements depend only on common materials parameters $\left(\omega_{\mathrm{po}}, \epsilon_{\mathrm{s}}, \epsilon_{\infty}\right.$, etc.) that can be calculated relatively inexpensively. Crucially, many of these properties are already tabulated in databases such as the Materials Project ${ }^{62}$ or can be obtained through relatively cheap ab initio calculations. Furthermore, the matrix elements can be evaluated in a fixed time regardless of the number of atoms in the system, and multiple temperatures and carrier concentrations can be calculated simultaneously with only a modest increase in the computational time. Full-timing information for the calculation of all firstprinciples inputs required to compute the transport properties of the materials discussed in this work and the scaling performance of each code routine is given in the Supplementary Methods.

Analysis of scattering rates and electron mobility. In Fig. 1, we compare mode-dependent scattering rates for $n$-Si and $n$-GaAs calculated by our method against fully first principles calculations (DFPT + Wannier) at $300 \mathrm{~K}$ obtained using the EPW and PERTURBO softwares 32,35 . The scattering of electrons in $\mathrm{Si}$ is dominated by acoustic phonons whereas polar optical phonon 

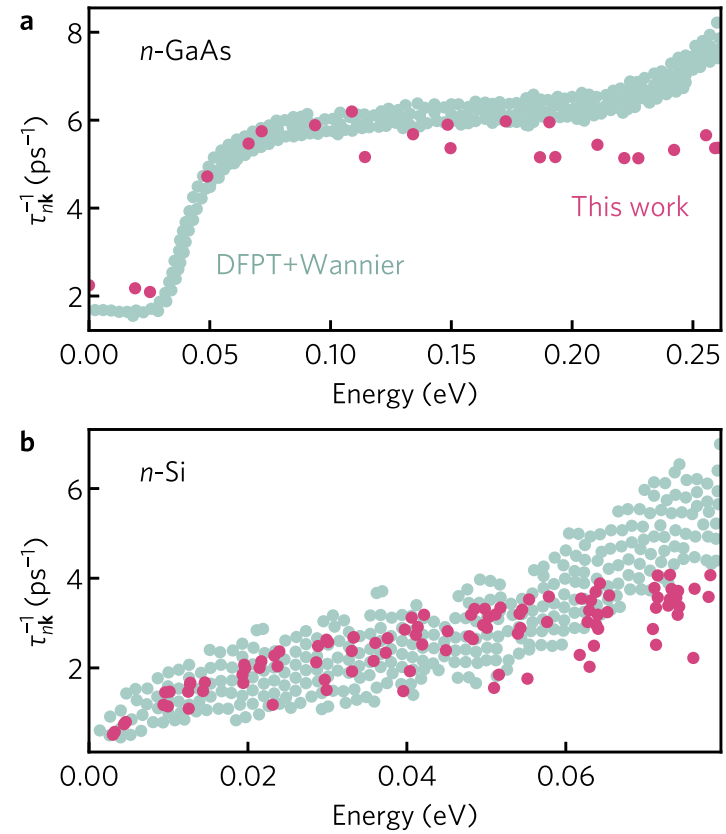

Fig. 1 Calculated scattering rates. Comparison of the calculated (pink) scattering rates, $\tau_{n \mathbf{k}}^{-1}$, against those obtained using density functional perturbation theory combined with Wannier interpolation (DFPT + Wannier, light teal) for (a) $n-\mathrm{GaAs}^{44}$ and (b) $n-\mathrm{Si}^{32}$ at $300 \mathrm{~K}$.

scattering dominates in GaAs, as revealed by the mobility analysis in Supplementary Fig. 9. Excellent agreement is seen for both systems, with the onset of polar optical emission scattering in $\mathrm{GaAs}$ well described by our calculations. The high degree of agreement for $\mathrm{Si}$ is somewhat surprising considering our calculations do not include optical phonons that are known to contribute to scattering at room temperature. However, as we do not consider symmetry selection rules in our calculations, the acoustic phonon scattering rate will be slightly overestimated and is expected to partially pick up some of the missing optical deformation scatterings. Whereas in $\mathrm{Si}$, group theoretical rules dictate that intervalley $g$-phonon transitions are only possible through longitudinal optical phonons, $\operatorname{Rode}^{63}$ and others ${ }^{64}$ have demonstrated the temperature-dependent transport behavior can reproduced considering only acoustic phonons if selection processes are ignored. Additional comparisons against DFPT + Wannier scattering rates for $3 \mathrm{C}$-SiC and $p$-SnSe are provided in Supplementary Fig. 13. In both cases, the shape and magnitude of the scattering rates is well reproduced, particularly at low energies, despite the simpler approach that does not involve an expensive DFPT calculation to obtain the matrix elements.

In Fig. 2, we compare the time taken to compute the transport properties of $\mathrm{NbFeSb}$ and $\mathrm{Ba}_{2} \mathrm{BiAu}$ (the full-timing breakdown is tabulated in Supplementary Tables 2 and 3). Taking into account the time required to compute all first-principles inputs and the electron mobility at a single temperature and carrier concentration, our method offers over a 2 order of magnitude speed up compared to DFPT + Wannier (an average of 29 core hours versus 8350 core hours). Considering only the time needed to obtain the scattering rates and transport properties (i.e., presuming all inputs have already been tabulated), our approach offers a 4 order of magnitude speed up (Fig. 2a). This can be exploited when performing calculations at multiple temperatures and carrier concentrations. For example, calculating the mobility of $\mathrm{Ba}_{2} \mathrm{BiAu}$ for 10 temperatures requires $~ 32,000$ core hours using DFPT + Wannier compared to $<35$ core hours with our approach (95\% of which is required to calculate the first principles inputs).
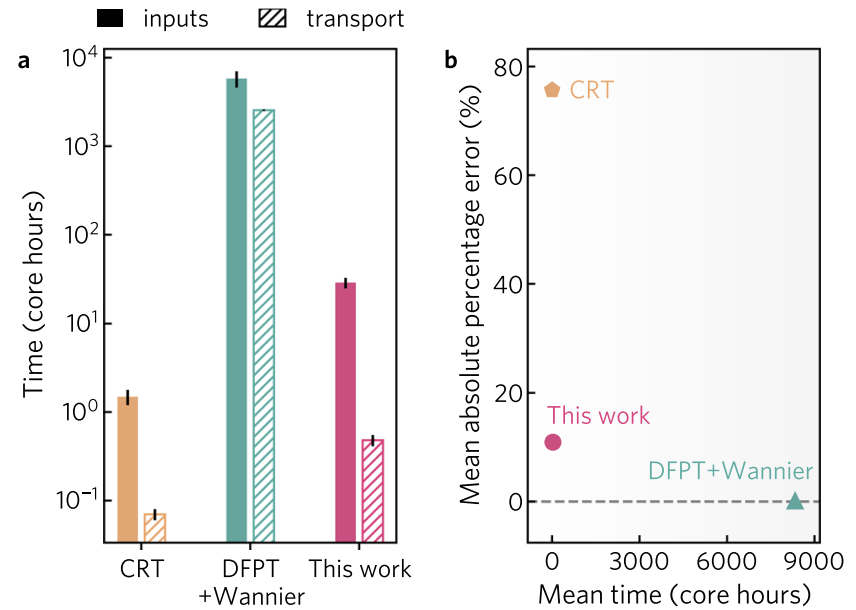

Fig. 2 Comparison of speed against accuracy for transport calculations. Existing methods for calculating electron transport properties are either computationally efficient but inaccurate (constant relaxation time, CRT, orange) or accurate but highly computationally demanding (density functional perturbation theory combined with Wannier interpolation, DFPT + Wannier, teal). The approach outlined in this work (pink) demonstrates accuracy comparable to state-of-the-art methods at $~ 1 /$ 500th of the computational cost. a The time required to obtain electron mobility for each method is broken down by the time spent computing first-principles inputs and performing the scattering and transport calculations. b The mean absolute percentage error in the calculated mobility at $300 \mathrm{~K}$ is compared to the total computational time (including the time to obtain all first-principles inputs). Results are averaged for NbFeSb ( $p$-type, $n=2 \times 10^{20} \mathrm{~cm}^{-3}$, DFPT + Wannier ${ }^{39,90}$ ) and $\mathrm{Ba}_{2} \mathrm{BiAu}$ ( $n$-type, $n=1 \times 10^{14} \mathrm{~cm}^{-3}$, DFPT + Wannier ${ }^{91}$ ). In (b), the mobility error is referenced with respect to state-of-the-art DFPT + Wannier calculations as high-quality experimental data were not available. The full timing breakdown for each material is provided in Supplementary Tables 2 and 3. Constant relaxation time calculations were performed with $\tau=10 \mathrm{fs}$.

Furthermore, we expect the relative cost advantage of our method to increase with system size as unlike in DFPT + Wannier the computational expense of the matrix elements does not depend on the number of atoms. This reduction in computational time, combined with similar accuracy to DFPT + Wannier [within $10 \%$, see Fig. 2 b], makes our approach amenable to the large-scale calculation of electronic transport properties.

Figure $3 \mathrm{a}$ plots the calculated mobility of $\mathrm{GaN}$ against experimental measurements, indicating very close agreement from 150 to $500 \mathrm{~K}$. As each scattering mechanism is treated with a separate matrix element, this allows the impact of individual scattering processes to be assessed. At low temperatures, the mobility of $\mathrm{GaN}$ is limited by impurity scattering, with polar optical phonon scattering dominating above $300 \mathrm{~K}$, as illustrated by the dashed lines in Fig. 3a. The total mobility taking into account all scattering mechanisms reproduces the experimental mobility with very high agreement. Further insight into the competing nature of the scattering mechanisms is provided by the energy dependence of the electron lifetimes and the resulting spectral conductivity, $\Sigma(\varepsilon)=v(\varepsilon)^{2} \tau(\varepsilon) N(\varepsilon)$ where $N$ is the density of states and $v$ is the group velocity, computed at $300 \mathrm{~K}$ and an electron concentration of $5.5 \times 10^{16} \mathrm{~cm}^{-3}$ (Fig. $3 \mathrm{~b}, \mathrm{c}$ ). Impurity scattering dominates at the conduction band edge but diminishes quickly as energy increases. At energies above $\omega_{\text {po }}$ of the band minimum (above the phonon emission threshold), polar-optical interactions are two orders of magnitude stronger than any other competing mechanism and act as the primary limiting factor for 

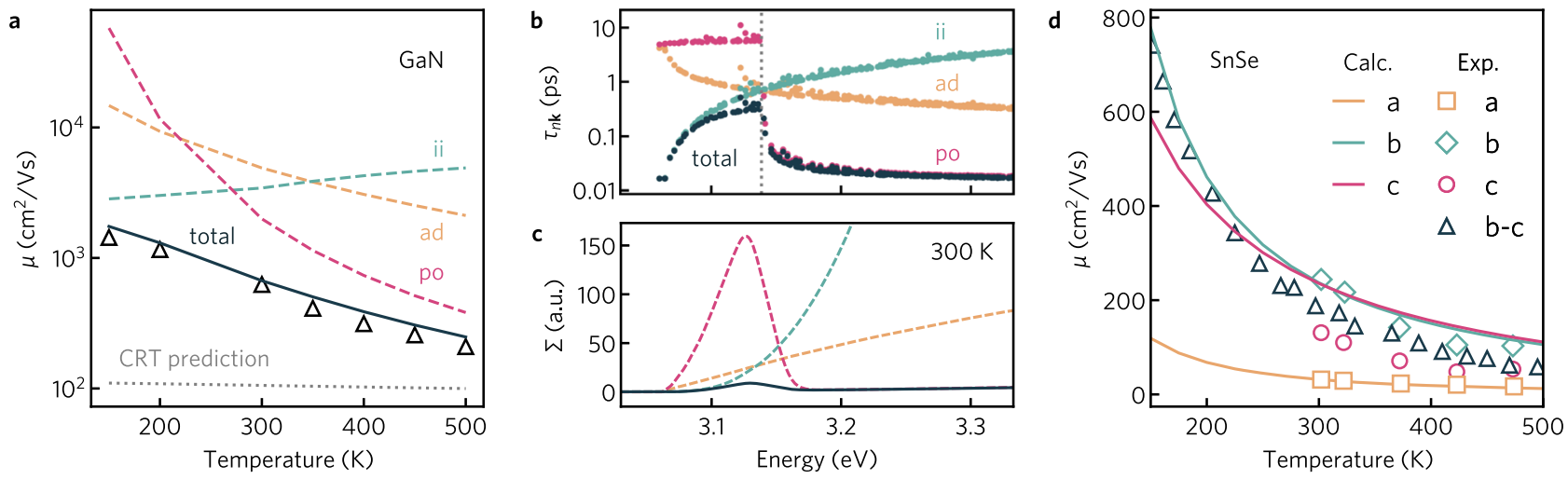

Fig. 3 Temperature-dependent transport properties of GaN and SnSe. a Comparison of the electron mobility, $\mu$, of GaN against experiment (black triangles, ${ }^{92}$ ). Mobility limited by ionized impurity (teal, ii), acoustic deformation potential (orange, ad), and polar optical phonon scattering (pink, po) is indicated in dashed lines. Total mobility taking into account all scattering mechanisms $\left(1 / \tau_{n \mathbf{k}}^{\mathrm{ii}}+1 / \tau_{n \mathbf{k}}^{\mathrm{ad}}+1 / \tau_{n \mathbf{k}}^{\mathrm{po}}\right)$ is given by the black solid line. Constant relaxation time (CRT) calculations with $\tau=10 \mathrm{fs}$ is given by dotted gray line. $\mathbf{b}$ Electron lifetimes, $\tau_{n \mathbf{k}}$ and (c) spectral conductivity, $\Sigma$, arising from different scattering processes in $\mathrm{GaN}$ at $300 \mathrm{~K}$. The valence band maximum is set to zero $\mathrm{eV}$. In (b), the vertical dotted gray line indicates the energy of the effective polar phonon frequency, $\omega_{\mathrm{po}}$. d Comparison of the direction-dependent mobility of SnSe against experiments $-a$ (orange), $b$ (teal), $c$ (pink) points from ref. ${ }^{66}, b-c$ (black) points from ref. ${ }^{93}$.

electron mobility, in agreement with the experimental findings of ref. 65 and DFPT + Wannier calculations ${ }^{42}$. In contrast, the mobility calculated using a constant relaxation time of $\tau=10 \mathrm{fs}-$ a value on the higher end of that typically employed in screening studies $^{25,26,28}$ - underestimates the mobility by a factor of $2-10$ depending on the temperature, as shown in Fig. 3a. More fundamentally, the CRTA does not reproduce the correct shape of temperature dependence as depicted in Fig. 3c. The ability of our method to reproduce the qualitative temperature dependence of transport properties, as well as make good approximations of quantitative behavior (often closely in-line with more detailed theoretical methods), thus represents a major advance for improving the accuracy of high-throughput methods.

A primary goal of the present approach is to extend wellestablished scattering matrix elements that were formulated for isotropic materials properties to be compatible with highly anisotropic materials. To that end, we have calculated the direction-dependent hole mobilities of Pnma structured SnSe at a carrier concentration of $3 \times 10^{17} \mathrm{~cm}^{-3}$, with the results compared to Hall measurements in Fig. 3d. Single-crystal SnSe has recently attracted significant attention as a thermoelectric material. Due to its layered structure, SnSe exhibits anisotropic transport properties, with the highest thermoelectric performance observed along the $b$ axis $^{66}$. Our calculations reproduce the strong directional dependence in transport measurements, in which the mobility parallel to the layers (along $b$ and $c$ ) is almost an order of magnitude larger than that perpendicular to the layers (along $a$ ). Our mobility results agree remarkably well with the considerably more computationally expensive electron-phonon calculations performed using DFPT +Wannier and $G_{0} W_{0}$ band structures ${ }^{41}$ (Supplementary Fig. 8). We note that additional anisotropy in the mobility between the $b$ and $c$ directions has been observed in high-temperature experimental measurements ${ }^{66}$. In both our calculations and DFPT + Wannier, however, the mobility along $b$ and $c$ are almost the same for temperatures above $300 \mathrm{~K}^{41}$. The discrepancy against the experiment is thought to derive from the use of a Hall factor $r_{H}$ of unity when extracting the carrier concentrations needed to compute mobility ${ }^{41}$ (further analysis is provided in the Supplementary Discussion). As we demonstrate in Supplementary Fig. 7, access to band and $\mathbf{k}$-dependent lifetimes can further be used to calculate electron linewidths that are qualitatively comparable to those measured through techniques such as angleresolved photoemission spectroscopy (ARPES) ${ }^{67}$.

Electron mobility and Seebeck coefficient across many systems. To demonstrate the generality of our approach, we investigate the transport properties of 21 semiconductors ranging from 2 to 48 atoms in their primitive unit cells. To highlight the compatibility of the method with high-throughput computations, all inputs (eigenvalues, wave functions, materials parameters) are obtained from density functional theory (DFT) using low-cost exchangecorrelation functionals (see the "Methods" section). All such materials parameters are listed in Supplementary Table 4. Results are compared to transport measurements on high purity singlecrystalline samples to minimize the effects of grain boundaries and crystallographic defects. Further details on the calculation methodology and selection of reference data are provided in the Supplementary Methods. The materials span multiple chemistries, doping polarities, and band structure types including anisotropic and multiband systems, and comprise: (i) conventional semiconductors, $\mathrm{Si}, \mathrm{GaN}, \mathrm{GaP}, \mathrm{GaAs}, \mathrm{InP}, \mathrm{ZnS}, \mathrm{ZnSe}, \mathrm{CdS}$, CdSe, and $\mathrm{SiC}$; (ii) the thermoelectric candidates $\mathrm{SnS}$, SnSe, $\mathrm{PbTe}$, $\mathrm{Bi}_{2} \mathrm{Te}_{3}$, and BiCuOSe; (iv) photovoltaic absorbers $\mathrm{PbS}$ and $\mathrm{CdTe}$; and (iii) transparent conductors, $\mathrm{SnO}_{2}, \mathrm{ZnO}$, and $\mathrm{CuAlO}_{2}$. Our dataset also includes the relatively complex $\mathrm{CH}_{3} \mathrm{NH}_{3} \mathrm{PbI}_{3}$ hybrid perovskite containing 48-atoms. In Fig. 4a we compare calculated mobility against experimental measurements for all 21 materials in our dataset. Calculations were performed using the experimentally determined carrier concentrations at a temperature of $300 \mathrm{~K}$. Results regarding the temperature and carrier concentration dependence of mobility for all materials (calculated, experimental, and comparison with CRTA) is provided in Supplementary Figs. 8 and 9 and include the breakdown of mobility by scattering type. These plots represent a comprehensive test of our approach, across many materials, not only at the single condition plotted in Fig. 4a but when conditions are varied.

The calculated mobilities agree closely with experiment across all materials, covering several orders of magnitude from $\mathrm{ZnO}$ $\left(180 \mathrm{~cm}^{2} / \mathrm{Vs}\right)$ to $n$-type GaAs $\left(\mu_{\text {exp }}=2.1 \times 10^{4} \mathrm{~cm}^{2} / \mathrm{Vs}\right)$. Notably, the calculated mobility (Spearman rank coefficient against experiment $\left.r_{\mathrm{s}}=0.93\right)$ improves significantly on results obtained using a constant relaxation time of $\tau=10 \mathrm{fs}\left(r_{\mathrm{s}}=0.52\right)$. As we 
a
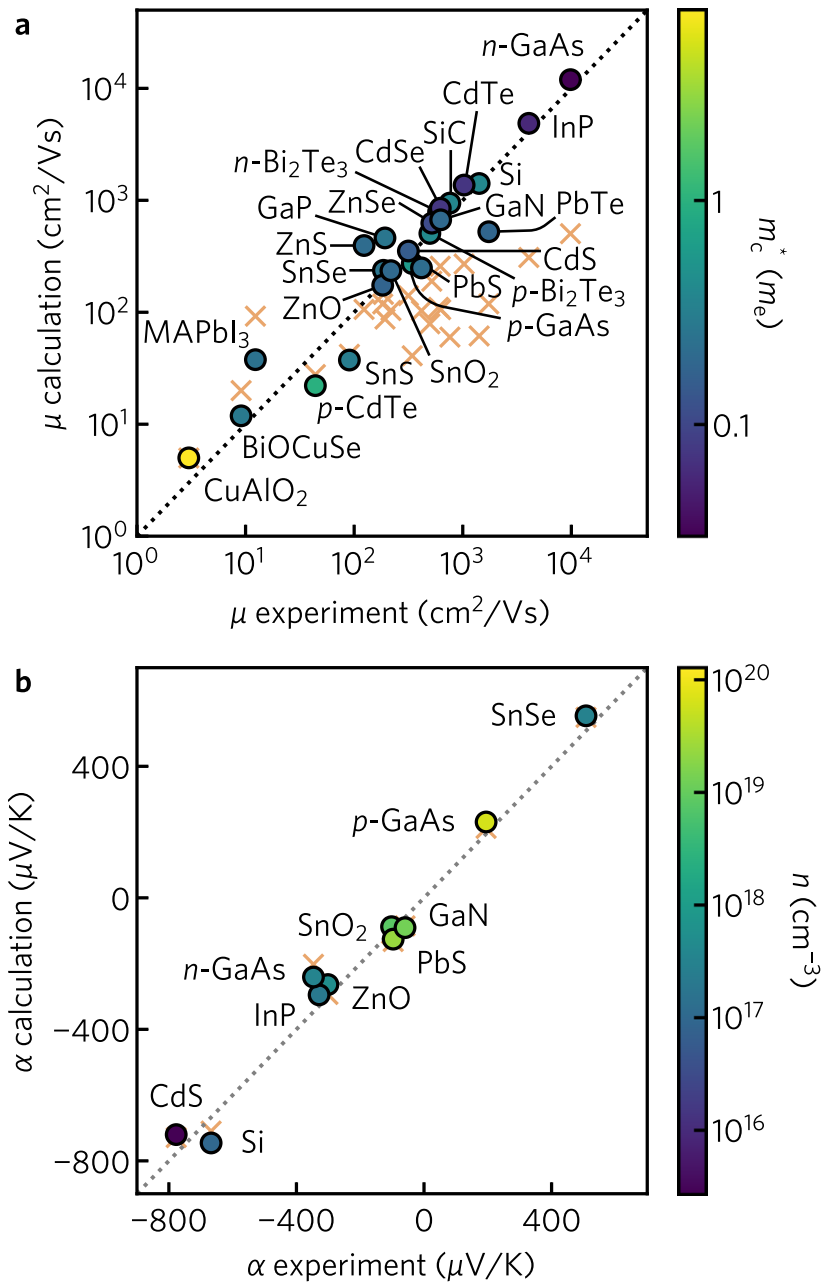

Fig. 4 Calculated mobility and Seebeck coefficient against experiment. a Comparison of carrier mobilities, $\mu$, at $300 \mathrm{~K}$ between calculations and experiments, with points colored by the conductivity effective mass $m_{c}^{*}$. b Comparison of Seebeck coefficients, $\alpha$, at $300 \mathrm{~K}$ between calculations and experiments, with points colored by the majority carrier concentration $n$. For Si and CdS we compare directly to the diffusive component of Seebeck coefficient only. $\mathrm{CH}_{3} \mathrm{NH}_{3} \mathrm{Pbl}_{3}$ has been abbreviated as $\mathrm{MAPbl}_{3}$. In (a) and (b), orange crosses indicate results computed using a constant relaxation time of $10 \mathrm{fs}$. Detailed temperature and carrier concentration results for each material are provided in Supplementary Figs. 8 and 11.

demonstrate in Supplementary Fig. 14, our method also improves the dependence of mobility on temperature, obtaining a mean squared error (MSE) of 0.20 consistent with DFPT + Wannier $(\mathrm{MSE}=0.19)$ and dramatically more accurate than a constant relaxation time $(\mathrm{MSE}=3.2)$. We note that more reasonable results can be obtained in the constant relaxation time approximation by fitting the lifetime to experimental measurements. However, in most cases, experimental measurements will not be available and thus this approach cannot easily be applied to new or uncharacterized systems. One alternative is to linearly scale the constant lifetime by the temperature according to $\tau_{T}=\tau \frac{300}{T} 68$. As demonstrated in Supplementary Figure 9, this results in a considerable improvement in the temperature dependence of mobility, reducing the mean squared error from 3.2 to 0.9 . By definition, however, this does not impact the mobility at $300 \mathrm{~K}$ and thus does not change the constant lifetime results presented in Fig. 4a.

Our approach demonstratesgreater deviation from experiment in materials with smaller mobilities such as $p-\mathrm{CuAlO}_{2}\left(3 \mathrm{~cm}^{2} / \mathrm{Vs}\right.$ in $a-b$ plane), where a local hopping mechanism is proposed to compete with band transport ${ }^{69}$, and $p$-CdTe in which spin-orbit coupling (SOC) is known to dramatically impact the scattering rates at the valence band edge ${ }^{70}$ but was not included in our calculations. Additional deviation is observed for $n-\mathrm{ZnS}$, where the calculated mobility is almost a factor of 4 larger than Hall measurements. We find this overestimation is largely due to the underestimation of the conduction band effective mass arising from the use of the PBE exchange-correlation functional $\left(m_{c}^{*}\right.$ PBE $\left.=0.16 m_{e}\right)$ when compared to experiment $\left(m_{c}^{*, \exp }=\right.$ $\left.0.22 m_{e}\right)^{71}$. As we detail in Supplementary Fig. 10, calculations performed using the hybrid HSE06 functional result in a larger effective mass $\left(m_{c}^{*, \mathrm{HSE}}=0.20 m_{e}\right)$ and improved agreement with the experimental mobility. Conversely, the mobility of $n$-type $\mathrm{PbTe}$ and $p$-type $\mathrm{SnS}$ are underestimated relative to the experiment. In both compounds, this originates from an overestimation of the effective mass. For example, in PbTe, HSE06 with the inclusion of spin-orbit coupling effects is required to reduce the effective mass from $m_{c}^{*}, \mathrm{PBE}=0.11 m_{e}$ to $m_{c}^{*, \mathrm{HSE}+\mathrm{SOC}}=$ $0.03 m_{e}$, in line with the experimental effective mass of $0.02 m_{e}$. In Supplementary Fig. 10, we demonstrate that more accurate treatment of the electronic structure significantly improves the agreement against the experiment across the full temperature range. Lastly, the deviation seen for $\mathrm{CH}_{3} \mathrm{NH}_{3} \mathrm{PbI}_{3}$ is likely due to the use of polycrystalline thin films in experimental measurements. As highlighted by Supplementary Fig. S8, our temperature-dependent results are in excellent agreement (within $5 \%$ at all temperatures) against fully first principles calculations performed using $\mathrm{EPW}^{37}$. The ability of our approach to accurately describe the electron-phonon coupling of a highly complex structure with 144 phonon-modes while remaining computationally efficient highlights its potential in highthroughput screening of transport behavior.

Accurate calculation of Seebeck coefficients is of primary interest in the prediction and analysis of thermoelectric materials. In Fig. $4 b$ we compare calculated Seebeck coefficients against those obtained experimentally at $300 \mathrm{~K}$. A comparison of the temperature dependence of the Seebeck coefficient for all materials is provided in Supplementary Fig. 11. We see reasonable agreement against experiment across the full range of materials, for both $p$ - and $n$-type samples, corresponding to positive and negative Seebeck coefficients, respectively. In Supplementary Fig. 12, we demonstrate that use of the HSE06 hybrid functional can further improve the agreement against the experiment for $n$ type GaAs. We note that for Si and CdS we compare directly to the diffusive component of Seebeck coefficient only, ignoring the effects of phonon drag which contribute substantially even at room temperature ${ }^{72-75}$. The Seebeck coefficient displays a weaker dependence on electron lifetimes than mobility and conductivity and so is often treated within the CRTA (in which case the specific relaxation time cancels in the equations). ${ }^{27}$. Figure $4 \mathrm{~b}$ indicates that this approximation is often justified due to the relatively small disagreements between constant relaxation time and mode-dependent relaxation time results, in-line with previous comparisons of CRTA against experimental data ${ }^{76}$.

\section{Discussion}

A key motivation in the development of the present approach is the opportunity to obtain accurate carrier lifetimes at minimal computational expense. Ideally, the method should be cheap enough to permit the calculation of transport properties for thousands of compounds in a high-throughput manner as well as large and complex materials. This would allow for reliable screening of materials for functional applications as well as enable investigations of systems with larger unit cells and more complex 
crystal structures. We stress that an expensive DFPT calculation is not required to obtain the matrix elements unlike methods such as $\mathrm{EPW}^{30}, \mathrm{PERTURBO}^{35}$, and EPIC STAR ${ }^{45}$. In our approach, the primary computational expense is the calculation of firstprinciples inputs, particularly the dielectric constant as detailed in Supplementary Table 2. However, due to our use of the relatively low-cost PBE exchange-correlation functional all inputs (electronic structure, $\Gamma$-point phonon frequencies, elastic constants, dielectric constants and piezoelectric tensor) can be obtained with moderate computational requirements (generally $<50$ core hours to compute all properties, see Supplementary Table 2). The calculation of transport properties takes even less time; the results for each material presented in this work were computed in under an hour on a personal laptop-further timing analysis, indicating the breakdown for different routines in the code, is presented in Supplementary Fig. 4. In addition, many of the materials properties required to calculate the scattering matrix elements are already available in computational materials databases. For example, at the time of writing the Materials Project contains over 3300 piezoelectric tensors, 7100 dielectric constants and phonon frequencies, and over 13,000 elastic constants ${ }^{62,77,78}$. Accordingly, our approach is well suited for the large-scale analysis of transport properties. To that end, we have made available a Python implementation of the method called $\mathrm{Ab}$ initio Scattering and Transport (AMSET) at https://github.com/hackingmaterials/ amset. Our goal is for this software to complement higher-level methods, such as EPW ${ }^{30}$ and PERTURBO ${ }^{35}$, which are state-ofthe-art but significantly more computationally demanding. A schematic overview of the package, indicating the inputs, outputs and command-line tools is given in Supplementary Fig. 3.

We stress that all electronic dispersions and wave functions were computed using the PBE functional which tends to overdelocalise electronic states and underestimate effective masses. In most cases, the calculated mobility is overestimated compared to the experiment, suggesting that the use of higher-level methods such as hybrid DFT or GW will be beneficial. In addition, there are several limitations of the current approach that may be addressed in a future release. In particular, optical deformation potential scattering is not treated, the symmetry of phonon modes is not used for filtering scattering events, and our matrix elements are not yet suitable for metals.

In conclusion, we introduced a method for calculating electron lifetimes and transport properties of semiconductors and insulators. Our method extends isotropic scattering matrix elements to support highly anisotropic materials and relies on a Brillouin zone integration scheme that overcomes the need for highly dense k-point sampling. The present approach offers similar accuracy to state-of-the art methods at $\sim 1 / 500$ th the computational cost and relies only on inputs that can be obtained from low-cost ab initio methods and that are routinely available in computational materials science databases. Furthermore, the agreement of mobility against experiment (Spearman rank coefficient $r_{\mathrm{s}}=0.93$ ) improves significantly on other low-cost methods such as a constant relaxation time approximation $\left(r_{s}=0.52\right)$ and temperature dependence is accurately captured. We expect that our method will enable accurate screening of transport properties in high-throughput computational workflows.

\section{Methods}

All DFT calculations were performed using the Perdew-Burke-Ernzerhof (PBE) exchange-correlation functional ${ }^{79}$ as implemented in the Vienna ab initio Simulation Package (VASP) ${ }^{80,81}$. Materials parameters, including elastic constants, dielectric tensors, deformation potentials, and phonon frequencies are listed in Supplementary Table 4 . Calculations were performed in a plane-wave basis set with scalar relativistic psueodpoentials and with the interactions between core and valence electrons described using the projector augmented-wave method $(\mathrm{PAW})^{82,83}$. The set-up, submission, and management of first-principles calculations were handled using the atomate workflow management software with the default parameters of version $0.83^{84,85}$. The plane-wave energy cutoff was set to $520 \mathrm{eV}$. Structure optimization was performed with a reciprocal k-point density of $64 \mathbf{k}$-points/§̊3. The uniform non-self-consistent calculations used as input to the scattering calculations were run with a reciprocal k-point density of $1000 \mathbf{k}$-points/ $\AA$ 3. Band gaps are corrected using a scissor operation to match those calculated by the Heyd-Scuseria-Ernzerhof (HSE06) hybrid functional ${ }^{86,87}$. Piezeoelectric constants, and static and high-frequency dielectric constants were computed using DFPT based on the method developed and by Baroni and Resta ${ }^{88}$ and adapted to the PAW formalism by Gajdoš et al. ${ }^{89}$. Elastic constants were obtained through the stress-strain approach detailed in ref. ${ }^{78}$. Spin-orbit interactions were included for calculations on $\mathrm{CH}_{3} \mathrm{NH}_{3} \mathrm{PbI}_{3}$ as they were necessary to obtain the correct band ordering at the conduction band minimum. A comparison of the experimental and HSE06 band gaps, along with initial and interpolated $\mathbf{k}$-point meshes are provided in Supplementary Table 5. All timing information (first-principles inputs and transport properties) displayed in Fig. 2a was obtained on a Intel Xeon Haswell processor E5-2698 v3 @ 2.3 GHz, except the EPW timing for NbFeSb. EPW timing information for $\mathrm{NbFeSb}$ was reported in ref. ${ }^{39}$ without specifying the processor type, so we have assumed a 1:1 correspondence in core performance.

\section{Data availability}

Source data are provided with this paper. Additional temperature-dependent datasets and input files generated and analysed in the current study are available from ref. ${ }^{94}$. Source data are provided with this paper.

\section{Code availability}

A Python implementation of the method called Ab initio Scattering and Transport (AMSET) is made available at https://github.com/hackingmaterials/amset, archived in ref. ${ }^{95}$, and provided as supplementary software 1.

Received: 8 December 2020; Accepted: 8 March 2021; Published online: 13 April 2021

\section{References}

1. Pimputkar, S., Speck, J. S., DenBaars, S. P. \& Nakamura, S. Prospects for LED lighting. Nat. Photonics 3, 180 (2009).

2. Green, M. A., Ho-Baillie, A. \& Snaith, H. J. The emergence of perovskite solar cells. Nat. Photonics 8, 506 (2014).

3. Fujishima, A. \& Honda, K. Electrochemical photolysis of water at a semiconductor electrode. Nature 238, 37 (1972).

4. Jain, A., Shin, Y. \& Persson, K. A. Computational predictions of energy materials using density functional theory. Nat. Rev. Mater. 1, 1 (2016).

5. Ellmer, K. Past achievements and future challenges in the development of optically transparent electrodes. Nat. Photonics 6, 809 (2012).

6. Snyder, G. J. and Toberer, E. S. Complex thermoelectric materials. Nat. Mater. 7, 105 (2008)

7. Bloch, F. Über die quantenmechanik der elektronen in kristallgittern. Z. Phys 52, 555 (1929).

8. Bardeen, J. \& Shockley, W. Deformation potentials and mobilities in nonpolar crystals. Phys. Rev. 80, 72 (1950).

9. Herring, C. \& Vogt, E. Transport and deformation-potential theory for manyvalley semiconductors with anisotropic scattering. Phys. Rev. 101, 944 (1956)

10. Harrison, W. A. Scattering of electrons by lattice vibrations in nonpolar crystals. Phys. Rev. 104, 1281 (1956a).

11. Meijer, H. \& Polder, D. Note on polar scattering of conduction electrons in regular crystals. Physica 19, 255 (1953).

12. Fröhlich, H. Electrons in lattice fields. Adv. Phys. 3, 325 (1954).

13. Xi, L. et al. Discovery of high-performance thermoelectric chalcogenides through reliable high-throughput material screening. J. Am. Chem. Soc. 140, 10785 (2018)

14. Yan, J. et al. Material descriptors for predicting thermoelectric performance. Energy Environ. Sci. 8, 983 (2015).

15. Wang, S., Wang, Z., Setyawan, W., Mingo, N. \& Curtarolo, S. Assessing the thermoelectric properties of sintered compounds via high-throughput $a b$ initio calculations. Phys. Rev. X 1, 021012 (2011).

16. Faghaninia, A., Ager III, J. W. \& Lo, C. S. Ab initio electronic gtransport model with explicit solution to the linearized Boltzmann transport equation. Phys. Rev. B 91, 235123 (2015).

17. Mandia, A. K., Muralidharan, B., Choi, J.-H., Lee, S.-C. \& Bhattacharjee, S. AMMCR: ab-initio model for mobility and conductivity calculation by using Rode Algorithm. Comput. Phys. Commun. 259, 107697 (2019).

18. Chaves, A. S., González-Romero, R. L., Meléndez, J. J. \& Antonelli, A. Investigating charge carrier scattering processes in anisotropic semiconductors through first-principles calculations: The case of $p$-type SnSe. Phys. Chem. Chem. Phys. 23, 900-913 (2020). 
19. Li, X. et al. TransOpt. A code to solve electrical transport properties of semiconductors in constant electron- phonon coupling approximation. Comput. Mater. Sci. 186, 110074 (2021).

20. Long, D. \& Myers, J. Ionized-impurity scattering mobility of electrons in silicon. Phys. Rev. 115, 1107 (1959).

21. Madsen, G. K. \& Singh, D. J. BoltzTraP. a code for calculating band-structure dependent quantities. Comput. Phys. Commun. 175, 67 (2006).

22. Madsen, G. K., Carrete, J. \& Verstraete, M. J. BoltzTraP2, a program for interpolating band structures and calculating semi-classical transport coefficients. Comput. Phys. Commun. 231, 140 (2018).

23. Pizzi, G., Volja, D., Kozinsky, B., Fornari, M. \& Marzari, N. BoltzWann: a code for the evaluation of thermoelectric and electronic transport properties with a maximally-localized Wannier functions basis. Comput. Phys. Commun. 185, 422 (2014).

24. Isaacs, E. B. \& Wolverton, C. Inverse band structure design via materials database screening: application to square planar thermoelectrics. Chem. Mater. 30, 1540 (2018)

25. Madsen, G. K. H. Automated search for new thermoelectric materials: the case of LiZnSb. J. Am. Chem. Soc. 128, 12140 (2006).

26. Bhattacharya, S. \& Madsen, G. K. H. A novel p-type half-Heusler from highthroughput transport and defect calculations. J. Mater. Chem. C 4, 11261 (2016).

27. Ricci, F. et al. An ab initio electronic transport database for inorganic materials. Sci. Data 4, 170085 (2017).

28. Miyata, M. et al. High-throughput screening of sulfide thermoelectric materials using electron transport calculations with OpenMX and BoltzTraP. J. Electron. Mater. 47, 3254 (2018).

29. Xing, G. et al. Electronic fitness function for screening semiconductors as thermoelectric materials. Phys. Rev. Mater. 1, 065405 (2017).

30. Poncé, S., Margine, E. R., Verdi, C. \& Giustino, F. EPW: electron-phonon coupling, transport and superconducting properties using maximally localized Wannier functions. Comput. Phys. Commun. 209, 116 (2016).

31. Agapito, L. A. \& Bernardi, M. Ab initio electron-phonon interactions using atomic orbital wave functions. Phys. Rev. B 97, 235146 (2018).

32. Poncé, S., Margine, E. R. \& Giustino, F. Towards predictive many-body calculations of phonon-limited carrier mobilities in semiconductors. Phys. Rev. B 97, 121201 (2018).

33. Gonze, X. et al. The abinit project: impact, environment and recent developments. Comput. Phys. Commun. 248, 107042 (2020).

34. Brunin, G. et al. Phonon-limited electron mobility in $\mathrm{Si}, \mathrm{GaAs}$, and $\mathrm{GaP}$ with exact treatment of dynamical quadrupoles. Phys. Rev. B 102, 094308 (2020).

35. Zhou, J.-J. et al. Perturbo: a software package for ab initio electron-phonon interactions, charge transport and ultrafast dynamics. Comput. Phys. Commun. 107970 (2021)

36. Ghosh, K. \& Singisetti, U. Ab Initio calculation of electron-phonon coupling in monoclinic $\beta-\mathrm{Ga}_{2} \mathrm{O}_{3}$ crystal. Appl. Phys. Lett. 109, 072102 (2016).

37. Poncé, S., Schlipf, M. \& Giustino, F. Origin of low carrier mobilities in halide perovskites. ACS Energy Lett. 4, 456 (2019a).

38. Li, W., Poncé, S. \& Giustino, F. Dimensional crossover in the carrier mobility of two-dimensional semiconductors: the case of InSe. Nano Lett. 19, 1774 (2019).

39. Samsonidze, G. \& Kozinsky, B. Accelerated screening of thermoelectric materials by first-principles computations of electron-phonon scattering. $A d v$. Energy Mater. 8, 1800246 (2018).

40. Poncé, S., Li, W., Reichardt, S. \& Giustino, F. First-principles calculations of charge carrier mobility and conductivity in bulk semiconductors and twodimensional materials. Rep. Prog. Phys. 83, 036501 (2020).

41. Ma, J., Chen, Y. \& Li, W. Intrinsic phonon-limited charge carrier mobilities in thermoelectric SnSe. Phys. Rev. B 97, 205207 (2018).

42. Poncé, S., Jena, D. \& Giustino, F. Hole mobility of strained GaN from first principles. Phys. Rev. B 100, 085204 (2019b).

43. Cao, J., Querales-Flores, J. D., Murphy, A. R., Fahy, S. \& Savić, I. Dominant electron-phonon scattering mechanisms in $n$-type $\mathrm{PbTe}$ from first principles. Phys. Rev. B 98, 205202 (2018)

44. Zhou, J.-J. \& Bernardi, M. Ab Initio electron mobility and polar phonon scattering in GaAs. Phys. Rev. B 94, 201201 (2016).

45. Deng, T. et al. EPIC STAR: a reliable and efficient approach for phonon- and impurity-limited charge transport calculations. npj Comput. Mater. 6, 46 (2020).

46. Rode, D. in Semiconductors and Semimetals, Vol. 10, pp. 1-89 (Elsevier, 1975).

47. Giustino, F., Cohen, M. L. \& Louie, S. G. Electron-phonon interaction using Wannier functions. Phys. Rev. B 76, 165108 (2007).

48. Giustino, F. Electron-phonon interactions from first principles. Rev. Mod. Phys. 89, 015003 (2017).

49. Khan, F. S. \& Allen, P. B. Deformation potentials and electron-phonon scattering: two new theorems. Phys. Rev. B 29, 3341 (1984).

50. Kartheuser, E. \& Rodriguez, S. Deformation potentials and the electronphonon interaction in metals. Phys. Rev. B 33, 772 (1986).
51. Resta, R. Deformation-potential theorem in metals and in dielectrics. Phys. Rev. B 44, 11035 (1991)

52. Auld, B. Acoustic Fields and Waves in Solids (Wiley Interscience Pub., 1973)

53. Zook, J. D. Piezoelectric scattering in semiconductors. Phys. Rev. 136, A869 (1964).

54. Born, M. Quantenmechanik der stoßvorgänge. Z. Phys. 38, 803 (1926).

55. Harrison, W. A. Mobility in zinc blende and indium antimonide. Phys. Rev. 101, 903 (1956b).

56. Verdi, C. \& Giustino, F. Fröhlich electron-phonon vertex from first principles. Phys. Rev. Lett. 115, 176401 (2015).

57. Sjakste, J., Vast, N., Calandra, M. \& Mauri, F. Wannier interpolation of the electron-phonon matrix elements in polar semiconductors: polar-optical coupling in GaAs. Phys. Rev. B 92, 054307 (2015).

58. Brooks, H. Scattering by ionized impurities in semiconductors. Phys. Rev. $\mathbf{8 3}$ 879 (1951).

59. Chattopadhyay, D. \& Queisser, H. Electron scattering by ionized impurities in semiconductors. Rev. Mod. Phys. 53, 745 (1981).

60. Graziosi, P., Kumarasinghe, C. \& Neophytou, N. Material descriptors for the discovery of efficient thermoelectrics. ACS Appl. Energy Mater. 3, 5913 (2020).

61. Onsager, L. Reciprocal relations in irreversible processes. I. Phys. Rev. 37, 405 (1931).

62. Jain, A. et al. Commentary: The Materials Project: a materials genome approach to accelerating materials innovation. APL Mater. 1, 011002 (2013).

63. Rode, D. L. Electron Mobility in Ge, Si, and GaP. Phys. Stat. Sol. (b) 53, 245 (1972).

64. Onton, A. Evidence of intervalley scattering of electrons in the extrinsic photoconductivity of n-type silicon. Phys. Rev. Lett. 22, 288 (1969).

65. Steigerwald, D. et al. III-V nitride semiconductors for high-performance blue and green light-emitting devices. Jom 49, 18 (1997).

66. Zhao, L.-D. et al. Ultralow thermal conductivity and high thermoelectric figure of merit in SnSe crystals. Nature 508, 373 (2014).

67. Park, C.-H. et al. Hove singularity and apparent anisotropy in the electronphonon interaction in graphene. Phys. Rev. B 77, 113410 (2008).

68. Graziosi, P., Kumarasinghe, C. \& Neophytou, N. Impact of the scattering physics on the power factor of complex thermoelectric materials. J. Appl. Phys. 126, 155701 (2019).

69. Kawazoe, H. et al. P-type electrical conduction in transparent thin films of $\mathrm{CuAlO}_{2}$. Nature 389, 939 (1997).

70. Yadav, D., Pauly, F. \& Trushin, M. Charge-carrier thermalization in bulk and monolayer CdTe from first principles. Phys. Rev. B 103, 125113 (2021).

71. Nagano, M., Kanie, H., Yoshida, I., Sano, M. \& Aoki, M. Photoluminescence in Sb-doped ZnS. Jpn. J. Appl. Phys. 30, 1915 (1991).

72. Herring, C. Theory of the thermoelectric power of semiconductors. Phys. Rev. 96, 1163 (1954).

73. Geballe, T. \& Hull, G. Seebeck effect in silicon. Phys. Rev. 98, 940 (1955)

74. Fiorentini, M. \& Bonini, N. Thermoelectric coefficients of $\mathrm{n}$-doped silicon from first principles via the solution of the Boltzmann transport equation Phys. Rev. B 94, 085204 (2016).

75. Morikawa, K. Seebeck effect in cadmium sulfide. J. Phys. Soc. Jpn. 20, 786 (1965)

76. Chen, W. et al. Understanding thermoelectric properties from highthroughput calculations: trends, insights, and comparisons with experiment. J. Mater. Chem. C 4, 4414 (2016).

77. De Jong, M., Chen, W., Geerlings, H., Asta, M. \& Persson, K. A. A database to enable discovery and design of piezoelectric materials. Sci. Data 2, 1 (2015).

78. de Jong, M. et al. Charting the complete elastic properties of inorganic crystalline compounds. Sci. Data 2, 150009 (2015).

79. Perdew, J. P., Burke, K. \& Ernzerhof, M. Generalized gradient approximation made simple. Phys. Rev. Lett. 77, 3865 (1996).

80. Kresse, G. \& Furthmüller, J. Efficient iterative schemes for ab initio totalenergy calculations using a plane-wave basis set. Phys. Rev. B 54, 11169 (1996a).

81. Kresse, G. \& Furthmüller, J. Efficiency of ab-initio total energy calculations for metals and semiconductors using a plane-wave basis set. Comput. Mater. Sci. 6, 15 (1996b).

82. Blöchl, P. E. Projector augmented-wave method. Phys. Rev. B 50, 17953 (1994).

83. Kresse, G. \& Joubert, D. From ultrasoft pseudopotentials to the projector augmented-wave method. Phys. Rev. B 59, 1758 (1999).

84. Mathew, K. et al. Atomate: a high-level interface to generate, execute, and analyze computational materials science workflows. Comput. Mater. Sci. 139, 140 (2017).

85. Mathew, K. et al. https://github.com/hackingmaterials/atomate/releases/tag/ v0.8.3 (2018).

86. Heyd, J., Scuseria, G. E. \& Ernzerhof, M. Hybrid functionals based on a screened Coulomb potential. J. Chem. Phys. 118, 8207 (2003).

87. Heyd, J., Scuseria, G. E. \& Ernzerhof, M. Erratum:"Hybrid functionals based on a screened Coulomb potential"[J. Chem. Phys. 118, 8207 (2003)]. J. Chem. Phys. 124, 219906 (2006). 
88. Baroni, S. \& Resta, R. Ab Initio calculation of the macroscopic dielectric constant in silicon. Phys. Rev. B 33, 7017 (1986).

89. Gajdoš, M., Hummer, K., Kresse, G., Furthmüller, J. \& Bechstedt, F. Linear optical properties in the projector-augmented wave methodology. Phys. Rev. B 73, 045112 (2006).

90. Zhou, J. et al. Large thermoelectric power factor from crystal symmetryprotected non-bonding orbital in half-Heuslers. Nat. Commun. 9, 1721 (2018).

91. Park, J., Xia, Y. \& Ozoliņš, V. High thermoelectric power factor and efficiency from a highly dispersive band in $\mathrm{Ba}_{2} \mathrm{BiAu}$. Phys. Rev. Appl. 11, 014058 (2019).

92. SuŁkowski, C. et al. Transport properties, specific heat and thermal conductivity of GaN nanocrystalline ceramic. J. Solid State Chem. 183, 2501 (2010).

93. Asanabe, S. Electrical properties of stannous selenide. J. Phys. Soc. Jpn. 14, 281 (1959).

94. Ganose, A. M. et al. Supporting data for "Efficient calculation of carrier scattering rates from first principles", https://doi.org/10.5281/zenodo.4574805 (2021a).

95. Ganose, A. M., Park, J., Faghaninia, A. \& Jain, A. Efficient calculation of carrier scattering rates from first principles. https://doi.org/10.5281/ zenodo.4574729 (2021b)

\section{Acknowledgements}

This work was intellectually led and funded by the U.S. Department of Energy (DOE), Office of Basic Energy Sciences, Early Career Research Program. Experimental data compilation was supported by DOE Basic Energy Sciences program - the Materials Project - under Grant No. KC23MP and the National Science Foundation (NSF) Graduate Research Fellowship under Grant No. DGE1106400 and DGE175814. This research used resources of the National Energy Research Scientific Computing Center, which is supported by the Office of Science of the U.S. Department of Energy under Contract No. DEAC02-05CH11231. Lawrence Berkeley National Laboratory is funded by the DOE under award DE-AC02-05CH11231. We acknowledge fruitful discussions with K. Inzani, Todd Karin for discussions regarding numerical integration, and Arsineh Apelian for assistance with deformation potential calculations.

\section{Author contributions}

All authors contributed to the conception and design of the study, as well as writing of the manuscript. A.M.G., J.P., and A.F. developed the software package and transport formalism with guidance from A.J. A.M.G. performed the transport calculations and analysed the results, with J.P. and A.J. contributing to the analysis. A.M.G., A.F., and R.W.-R. collated experimental and computational transport data. K.A.P. supervised the work performed by R.W.-R. A.J. supervised the study.

\section{Competing interests}

The authors declare no competing interests.

\section{Additional information}

Supplementary information The online version contains supplementary material available at https://doi.org/10.1038/s41467-021-22440-5.

Correspondence and requests for materials should be addressed to A.M.G. or A.J.

Peer review information Nature Communications thanks Neophytos Neophytou, Huijun Liu and the other, anonymous, reviewer(s) for their contribution to the peer review of this work.

Reprints and permission information is available at http://www.nature.com/reprints

Publisher's note Springer Nature remains neutral with regard to jurisdictional claims in published maps and institutional affiliations.

\section{(c) (i)}

Open Access This article is licensed under a Creative Commons Attribution 4.0 International License, which permits use, sharing, adaptation, distribution and reproduction in any medium or format, as long as you give appropriate credit to the original author(s) and the source, provide a link to the Creative Commons license, and indicate if changes were made. The images or other third party material in this article are included in the article's Creative Commons license, unless indicated otherwise in a credit line to the material. If material is not included in the article's Creative Commons license and your intended use is not permitted by statutory regulation or exceeds the permitted use, you will need to obtain permission directly from the copyright holder. To view a copy of this license, visit http://creativecommons.org/ licenses/by/4.0/.

(C) The Author(s) 2021 